\title{
High rate performance of Small-pad Resistive Micromegas. Results of different resistive protection concepts
}

\section{M. lodice ${ }^{* \dagger a}$, M. Alviggi ${ }^{b, c}$, V. Canale ${ }^{b, c}$, M. Della Pietra ${ }^{b, c}$, C. Di Donato ${ }^{b, d}$, E. Farina ${ }^{e}$, S. Franchino ${ }^{f}$, P. lengo $^{g}$, L. Longo ${ }^{h}$ F. Petrucci $^{a, i}$, E. Rossi ${ }^{a, i}$, G. Salamanna ${ }^{a, i}$, G. Sekhniaidze $^{b}$, O. Sidiropoulou ${ }^{g}$}

${ }^{a}$ INFN Roma Tre, Rome, Italy, ${ }^{b}$ INFN Napoli, Naples, Italy, ${ }^{c}$ Università di Napoli Federico II, Naples, Italy, ${ }^{d}$ Università di Napoli Parthenope, Naples, Italy, ${ }^{e}$ Università di Pavia and INFN Pavia, Pavia, Italy, ${ }^{f}$ Heidelberg University, Heidelberg, Germany, ${ }^{g}$ European Center for Nuclear Research, CERN, Geneva, Switzerland, ${ }^{h}$ INFN Sezione di Lecce and Dipartimento di Matematica e Fisica, Università del Salento, Lecce, Italy, ${ }^{i}$ Università di Roma Tre, Rome, Italy,

\begin{abstract}
We present the development of resistive micromegas aiming at precision tracking in high rate environment without efficiency loss up to several $\mathrm{MHz} / \mathrm{cm}^{2}$. To achieve this goal, the optimisation of the spark protection resistive layer and the miniaturisation of the readout elements, are key elements of the project.

Several small-pad micromegas detectors have been built with a anode plane matrix of $48 \times 16$ readout pads, rectangular in shape, with dimensions $0.8 \times 2.8 \mathrm{~mm}^{2}$. With this anode/readout layout, different concepts of the spark protection resistive layers have been implemented. Characterisation and performance studies of the detectors have been carried out by means of radioactive sources, $\mathrm{X}$-Rays, and test beam. A comparison of the performance obtained with the different resistive layout is presented, in particular focusing on the response under high irradiation and high rate exposure.
\end{abstract}

The 39th International Conference on High Energy Physics (ICHEP2018)

4-11 July, 2018

Seoul, Korea

\footnotetext{
* Speaker.

${ }^{\dagger}$ E-mail: mauro.iodice@roma3.infn.it
} 
Micromegas resistive detectors have already proven suitable for precision tracking in particle density environments up to few $\mathrm{kHz} / \mathrm{cm}^{2}$ (e.g. [1]). Rate capability up to tens $\mathrm{MHz} / \mathrm{cm}^{2}$ and low occupancy can be achieved by using few $\mathrm{mm}^{2}$ readout pads, and the optimisation of the spark protection resistive layer. Such a rate capability will be required in upgrades of forward muon detectors of LHC experiments as well as in experiments at future colliders. The anode segmentation in pads in the proposed detector, has been originally inspired by a similar project for the COMPASS experiment [2] and sampling calorimetry [3]. With respect to these developments, the present project aim at a reduction of pad-size from $\mathrm{O}\left(\mathrm{cm}^{2}\right)$ to $\mathrm{O}\left(\mathrm{mm}^{2}\right)$.

All Small-pad micromegas detectors presented in this paper consist of a similar anode plane, segmented with a matrix of $48 \times 16$ readout pads. Each pad with rectangular shape with a pitch of 1 and $3 \mathrm{~mm}$ in the two coordinates. The active surface is $4.8 \times 4.8 \mathrm{~cm}^{2}$ with a total number of 768 channels, routed off-detector for readout. The layout of the anode plane can be seen in Fig. 1-topleft. On top of this anode plane, different concepts of the spark protection resistive layer have been implemented.

The prototypes "series-1" are based on a pad-patterned layout with embedded resistors [4]. Each pad is totally separated from the neighbours. The anode pads are overlaid by resistive pads, both interconnected by intermediate "embedded" resistors, as shown in bottom-left of Fig. 1. The total resistance between the resistive and anode pads is in the range 3-7 MOhm.

The "series-2" implements a double uniform resistive layer with grounding connection vias. Each layer is produced by sputtering deposition of DLC (Diamond Like Carbon structure) on insulating (Kapton) foils. The two resistive layers are interconnected between them and to the readout pads with a network of conducting links with a few $\mathrm{mm}$ pitch, filled with silver past, to evacuate the charge, as sketched on the right part of Fig. 1. The detector was divided in two halves, each one having a different pitch of the conducting vias through the DLC layers: $6 \mathrm{~mm}$ and $12 \mathrm{~mm}$ respectively. This spark protection mechanism with a double DLC layer has been first implemented in $\mu$-RWELL detectors prototypes [5]. Most of the results presented here for series-2 detectors refers to a DLC sheet resistivity of about 50-70 MOhm.

The detectors finally are completed with a bulk Micromegas process [6], defining the 128 $\mu \mathrm{m}$ amplification gap with a metallic micro-mesh supported by insulating pillars, and with a drift cathode defining the $5 \mathrm{~mm}$ wide conversion gap.

The performance results of a series- 1 detector with patterned screen printed resistive layer have been published and reported at several Conferences (see e.g. [4]). The detectors are operated with a $\mathrm{Ar} / \mathrm{CO}_{2}$ 93/7 gas mixture, reaching amplification gains larger than $2 \times 10^{4}$ In terms of behaviour at high rates, this prototype provides very satisfactory results, still operating stably with a gain of 4000 up to $150 \mathrm{MHz} / \mathrm{cm}^{2}$ with a ${ }^{55} \mathrm{Fe}$ source. Some features are nevertheless observed: above $\mathrm{O}(100) \mathrm{kHz} / \mathrm{cm}^{2}$ dielectric charging up effects start to show-up, reducing the gain by about $20 \%$, and, above $10 \mathrm{MHz} / \mathrm{cm}^{2}$, the voltage drop contribution, due to the current flow through the pads resistive path, dominates the further reduction of gain.

To further improve the performance of small pad micromegas, the series-2 layout has been produced and tested. In this Section the results of the two types are compared. Series -1 and 2 will be referred to as "Embedded resistor - EMB" and "DLC" types, respectively.

In Fig. 2-Left, the gain curves as a function of the amplification voltage, for the two prototypes are reported. Data have been taken with a low $(1.3 \mathrm{kHz})$ and a high $(128 \mathrm{kHz})$ intensity ${ }^{55} \mathrm{Fe}$ 


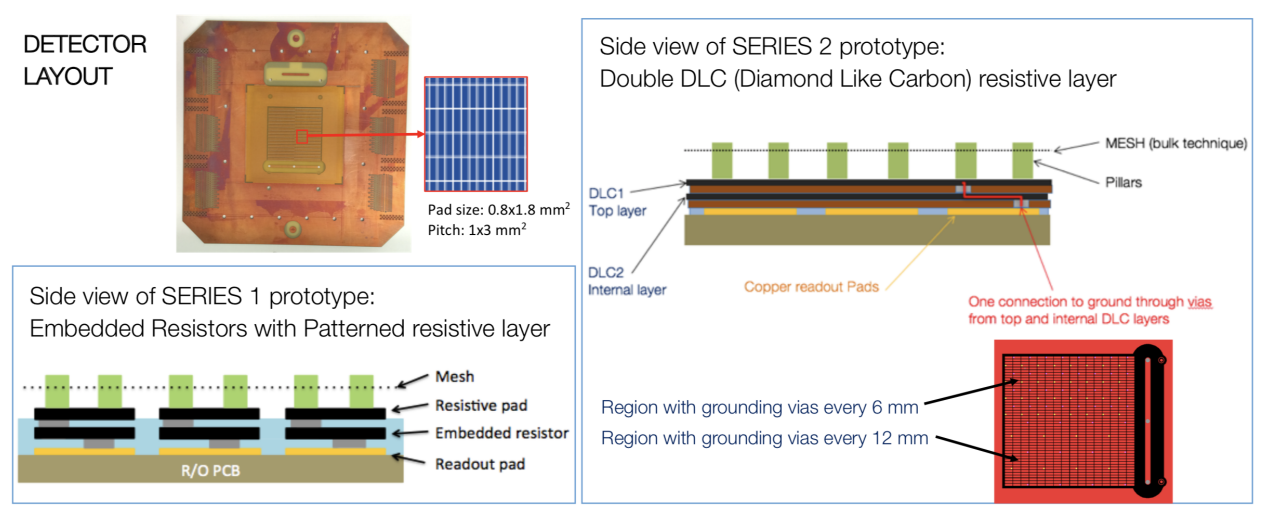

Figure 1: Top-left: Photo and Layout of the anode plane PCB; Bottom-Left: side view sketch of the "series1" embedded resistors prototypes; Right: side view sketch of the "series-2" DLC prototypes. Pillars and meshes are also sketched. The drift gap and cathode plane are not shown in the sketches.

radioactive source. While for the EMB prototype a reduction of about $20 \%$ with the high intensity source is observed, in the case of the DLC the gain measurements with the high and the low intensity sources perfectly overlap.
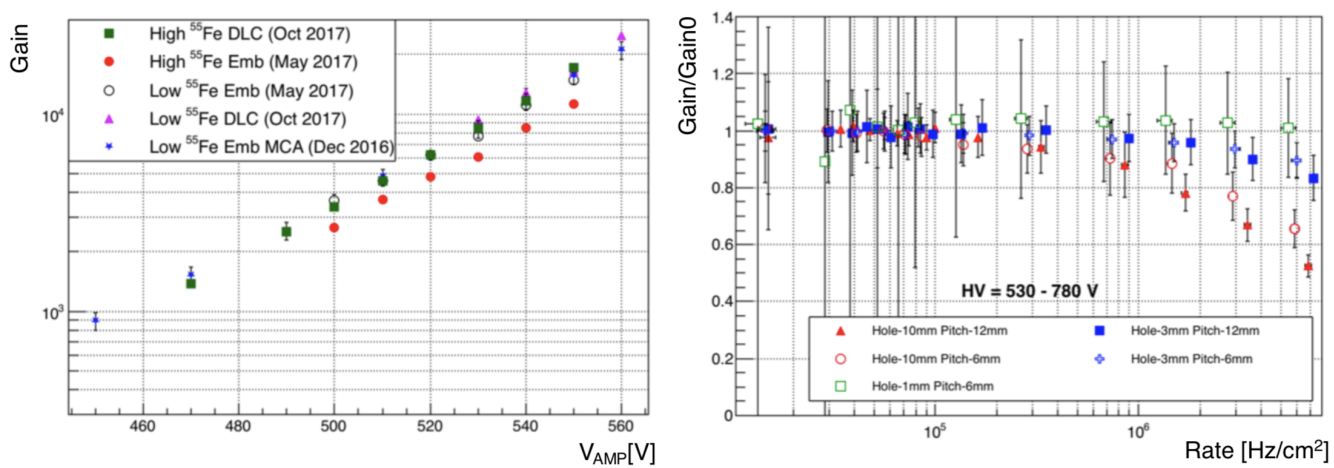

Figure 2: Left: Gain Vs HV for EMB and DLC prototypes. The gain was measured reading the detector current from the readout pads with a pico-ammeter and shown to be consistent with measurements of the ${ }^{55} \mathrm{Fe}$ peak position obtained with a multi-channel-analyser (MCA in the plot) from the signals taken from the mesh. Right: Relative gain of the DLC detector under high rate of X-rays.

In Fig. 3, the measured detector currents (proportional to the gain) as a function of time are shown. The left-panel reports the results for the EMB detector, taken at different values of the amplification voltage, irradiated with the low (red) and the high (black) intensity sources. A clear drop with time of about $25 \%$ is observed and saturating. This behaviour, together with the similar observed drop in gain from Fig. 2-left, is compatible with dielectric charge-up effect of kapton between pads. The right-panel of Fig. 3 is a very convincing proof that charge-up is absent for the DLC detector. A very stable behaviour is observed. No decrease with time, also at very high rates. This is indeed an expected behaviour since, due to the uniform DLC resistive layers, there is no exposed dielectric in the amplification gap (with the only exception of the pillars). 

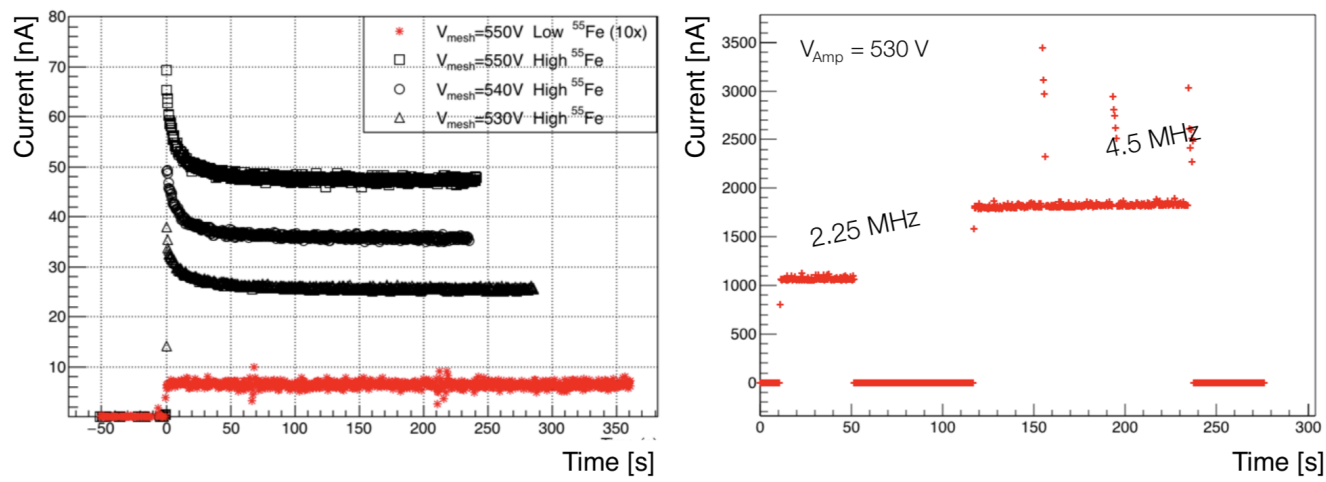

Figure 3: Current as a function of time with detectors intermittently exposed to radioactive sources

The behaviour of the DLC detector under high rate has been studied with a X-ray gun. In order to focus on the gain drop, the measurements as a function of the rate shown in Fig. 2-right have been normalised to the mean value of the gain measured at low rates (below $80 \mathrm{kHz} / \mathrm{cm}^{2}$ ). The amplification voltage was $530 \mathrm{~V}$ and the absolute, reference value of the gain was about 12000 . Data have been taken with different collimators $(1,3,10 \mathrm{~mm}$ in diameter) and irradiating different positions of the DLC detector: the region with grounding vias every $6 \mathrm{~mm}$ and $12 \mathrm{~mm}$. At rates as high as $10 \mathrm{MHz} / \mathrm{cm}^{2}$ a gain drop up to about $50 \%$ is observed with the large collimator $(10 \mathrm{~mm})$, The gain drop decreasing for smaller irradiation spots. This indicate that the resistivity of the foil ( 50-70 MOhm/sq) is not small enough to quickly evacuate the collected charge, thus causing a voltage drop of the anode with respect to the mesh, thus producing a lower amplification electric field. Moreover, gain drop slightly depends from the pitch of the grounding conducting vias. As expected the farther is the connection to the ground, the higher the resistance of connection the higher the voltage drop. Tests with DLC foils with lower resistivity ( $20 \mathrm{MOhm} / \mathrm{sq})$ are ongoing.

\section{References}

[1] ATLAS Collaboration, New Small Wheel Technical Design Report, CERN-LHCC-2013-006.

[2] F. Thibaud et al., Performance of large pixelised MicroMEGAs detectors in the COMPASS environment JINST 9 (2014) C02005

[3] M. Chefdeville et al., Resistive Micromegas for sampling calorimetry, a study of charge-up effects Nucl. Instrum. Meth. A 824 (2016) 510-511

[4] M. Alviggi et al, Small-pads resistive micromegas JINST 12 (2017) C03077 M. Alviggi et al, Construction and test of a small-pad resistive Micromegas prototype ACCEPTED for publication by JINST M. Alviggi et al., Small-pad resistive Micromegas for high rate environment: Performance of different resistive protection concepts Nuclear Inst. and Methods in Physics Research, A In Press; https://doi.org/10.1016/j.nima.2018.10.052

[5] G. Bencivenni et al., The $\mu$-RWELL detector JINST 2 (2017) C06027

[6] I. Giomataris et al., Micromegas in a bulk, Nucl. Instrum. Meth. A 560 (2006) 405 